\title{
La geometria in Italia, dal Cremona ai giorni nostri.
}

\author{
Prolusione al Corso di geometria superiore \\ letta dal prof. Bentamino Segre a Bologna il 13 gennaio 1982.
}

È per me sommo onore l'esser stato chiamato a far parte di questa gloriosa Università, che vanta origini e tradizioni splendide - studiate con profondità ed acume, per ciò che concerne le matematichè, dal nostro illustre Collega BontonotT - e che ancor oggi conta fra i suoi membri uomini eminenti, di fama mondiale. Ed è con commozione non scevra da titubanza che salgo su questa cattedra, che vide il sorger radioso di quell'astro che fn Luigi Cremona, non a torto detto il Padre della moderna geometria ita* liana, e sulla quale si son succeduti uomini illustri, quali Pretro Bosohi, Domenico Montesano, Federigo Enriques ed Enrioo Bompiani.

Mi sorregge però lo sviscerato amore per la scienza, che han saputo infondermi i venerati maestri ConRado SEgre e Francesco Severi - ai quali, particolarmente in questo istante, il mio pensiero si volge con riconoscenza - e la ferma volontà di fare quanto è in me per non venir meno al buon nome di Bologna e della scienza italiana.

Non mi nascondo tutta l'entità di questo proposito, per apprezzar giustamente il quale è d'uopo aver presente il prodigioso sviluppo che ha avuto in Italia la matematica, e più specialmente la geometria, da CREMoNA ai giorni nostri; ed ora appunto - con rapido schizzo - mi accingo a cogliere i tratti più salienti di questo periodo, che ci ha condotti ad un primato indiscusso, che le altre Nazioni ci invidiano.

Quando nel 1860 il trentenne CRemona, chiamato dalla fiducia di TERENzro Mamrant, saliva sulla cattedra di geometria superiore, allora fondata in Bologna dal dittatore FARINI, le condizioni della geometria in Italia - che pur nei secoli precedenti aveva avuto valenti cultori - erano, fatte poche eccezioni, piuttosto modeste. In Francia ed in Germania, invece, da oltre un cinquantennio, una eletta e numerosa schiera di Geometri andava costruendo quel superbo edificio - degno di reggere il confronto colla immortale costruzione di EUChIDE - che è la geometria proiettiva. 
Le ragioni del nostro assenteismo da questo fervore di ricerche e di studi, son da imputarsi alle disgraziate condizioni politiche di quel periodo della nostra storia, per cui le varie regioni d'Italia erano fra loro divise e rette da governi - in maggior parte stranieri o mancipì dello straniero - che. nell' elevazione scientifica e calturale dei singoli e delle masse, vedevano soltanto un pericolo alla loro stabilità. Si che, ad esempio, in occasione del VII ${ }^{\circ}$ Con. gresso degli Scienziati italiani tenutosi in Napoli nel 1845, ed al quale parte* cipavano molti di fuori, il re Ferdnando II $^{\circ}$ ingiungeva ad un sno ministro di «tenergli d'occhio quei pennaiuoli».

Per dare un'idea del modo curioso con cui a quei tempi renivano dai governanti considerate le cose universitarie, riferirò col VoLTERRA il seguente aneddoto (1). Essendosi rese vacanti verso il 1835, in un' università toscana, le cattedre di diritto ecclesiastico e di algebra, due cultori di queste materie chiesero ed ottennero di ricoprirle. Però, nell' assegnarle, esse furono per errore scambiate. Le proteste per la rettifica non valsero a nulla e non si vollero cambiare i «mutupropri» granducali di nomina, già firmati: il matematico rinunziò allora ad inseguare il gius canonico, ma il giurista insegnò algebra per tutta la vita.

All'ignavia dei governi occorre poi aggiungere un'altra circostanza, che - pur provando la maturità a cui erano giunti gli Italiani - non poteva certo favorire quella calma che è così necessaria per lo svolgersi proficuo degli studi. Voglio alludere al generoso fermento che accomunava studenti e docenti nelle ideologie di patria e di libertà, e nelle lotte contro lo straniero oppressore. E, fra gli esempi più tipici, ricorderò a questo riguardo che nel 1848 il CREMona, interrotti gli studi, era accorso volontario in ainto di Milano insorta, combattendo quindi a Nervesa, alla Piave, a Treviso, e partecipando infine alla difesa di Venezia; e che nello stesso anno il Mossomt, professore di meccanica razionale a Pisa, aveva combattuto a Curtatone e Montanara col battaglione universitario.

In tali condizioni può anzi destar meraviglia che in Italia le tradizioni scientifiche non fosser per anco morte del tutto, e che - non ostante i molti ostacoli - vi fossero uomini aperti alle nuove correnti. "Da molto tempo » dice il Cremoxa nella celebre Prolusione da lui tenuta salendo su questa cattedra $\left({ }^{2}\right)$ «nelle università d'Italia non si poterono insegnare fuor che $i$ «primi rudimenti delle scienze esatte; ed i buoni ingegni ne uscivano questo «solo sapendo, esistere vaste e meravigliose dottrine di cui era lor noto ap-

(1) Cfr. "Atti del IV Congresso internazionale dei matematici " (Roma, 1908), t. I, p. 57.

(2) Ved. Opere matematiche di L. Crearons, t. I, p. 238. 
«pena l'alfabeto. Se non che ove cessava la scuola, soccorreva talvolta l'opera "generosa d'alcun professore; che con consigli, con libri, con eccitamenti, "indirizzava i giovani a quegli studi che non si eran potuti fare nella pub. «blica senola».

Ed ecco infatti a Napoli, ove era vissuto Nicora Fergola (1753-1822), sorgere una numerosa Scuola geometrica che contava fra i migliori Vinoenzo FLAUTI (1782-1863), la quale perseguiva con poohe varianti i metodi sintetici degli antichi; in contrapposto Fortunato Padula (1816-1881) e Nicola Trudi (1811-1884) sostenevano 1'opportunità dell'impiego dei metodi analitici, senza giungere però ad una concezione veramente elevata della nuova geometria. Altri distinti cultori della geometria analitica erano gli abati Domentco CHELini (1802-1878) e Barnaba Tortolini (1808-1874) a Roma, e Givsto BeL. LAVITIS (1803-1880) - uno dei creatori del moderno calcolo vettoriale - a Padova; infine fra i centri più importanti di studi matematici in Italia vi era allora Pavia, ove insegnavano Gaspare MaInaRd (1800-1879), ANTonio Bordont (1788-1860) e Francesco Brioschi (1824-1897), che doveva poi affer* marsi come uno dei più grandi algebristi del secolo.

Questi pochi ricercatori avevano fra loro contatti quasi nulli, ed a loro poteva solo giungere affievolita l'eco delle mirabili scoperte che si venivano facendo all'estero, ogni relazione essendo estremamente difficile e - dati i tempi - pericolosa. Una notevole influenza deve, da questo punto di vista, aver avuto il viaggio in Italia compiuto nel 1843-44 da SteIneR, JACoBI, DIRICHLET e BORCHARDT, e specialmente la loro permanenza a Roma ed a Napoli. Ad ogni modo le difficoltà che incontravano allora in Italia il progredire ed il diffondersi della seienza, erano gravissime: non ultima quella - a cui già si è accennato - della mancanza di cattedre da dove le nuove verità potessero venir bandite. Dobbiamo quindi esser sommamente grati all'opera di quei solitari, cui non muoveva la speranza di onori o di ricom. pense, ma solo l'amore disinteressato per la scienza.

Con l'avvento del governo nazionale, venivano nel 1860 create due cattedre di geometria superiore a Bologna ed a Napoli, rispettivamente ricoperte da Luigi Cremona e da Giuseppe Battaglini; e non tardavano a manifestarsi le benefiche conseguenze di questi provvedimenti, e delle mutate condizioni politiche.

L'opera del Cnemora nella geometria algebrica - per dirla col CASTELNuovo $\left({ }^{3}\right)$ - «chiude un'epoca per aprirne una nuova». È infatti col CRE-

(3) Crr. "Rendiconti R. Acc. Naz. dei Lincei", serie VI, t. 12 (1930), p. 613. 
INONA che la geometria proiettiva raggiunge le mete più eccelse, pervenendo alla teoria generale delle curve e delle superficie algebriche; e, d'altro canto, la scoperta fondamentale delle trasformazioni cremoniane erea tutto un nuovo ordine di ricerche importantissime - non per anco esanrite - al quale appartengono i più brillanti risultati della Scuola geometrica italiana nell' ultimo cinquantennio.

La geometria proiettiva, sorta quasi miracolosamente dall'antica, principalmente ad opera di Desargues e Pascal, si era sviluppata erigendosi a dottrina solo circa due secoli dopo, con Monge, Carnot, Lamé, Ponoeteet, Gergonne, Bobiluier, Moebius, Steiner, Chasles, Pluecker, mediante l'abile contemporaneo sfruttamento della geometria degli antichi e dello strumento analitico, che era stato forgiato per altri scopi nel secolo XVII ${ }^{\circ}$ da Fermat e Descartes; finchè nel 1847 voN Staddi le permette di assurgere a scienza autonoma, creando un'opera di imperitura bellezza e di eleganza inimitabile. È questa la scienza a cui il Cremona subito si dedica con entusiasmo, prendendola ad argomento del sno Corso di geometria superiore: e si deve in gran parte all'opera scientifica e didattica del Cremona se essa oggi ci appare assai più elementare, tanto da trovar posto fra i Corsi propedeutici, e se inoltre - grazie alle numerose applicazioni alla geometria descrittiva ed alla statica grafica - essa insegnasi anche agli allievi ingegneri.

I metodi puristi dello STAUDT, se per armonia e rigore posson reggere il paragone con quelli di Eucurde, non si prestan che con difficolta agli sviluppi alteriori della teoria: cio intui il CREMoNA, che seppe maestrevolmente unirli ad alcuni prineipi fondamentali tratti dall'algebra, creando - per così dire - una nuova algebra vivificata da schietto spirito geometrico. Il connubio fra algebra e geometria non era nuovo nella storia ( $\left.{ }^{4}\right)$, ed anzi esisteva assai prima della scoperta della geometria analitica; ma è solo con CrewoNA ch' esso assume una forma caratteristica, in cui possono riconoscersi il rigore e la generalità dell'algebra fusi coi preziosi suggerimenti pôrti dall'intuizione geometrica. A questo indirizzo appartengono le ormai classiche ricerche del Cremona indicate poc'anzi, relative allo studio proiettivo delle curve (1862) e delle superficie algebriche (1866-67).

Nella geometria proiettiva hanno grande importanza le cosi dette trasformazioni proiettive: è p. es. proiettiva la corrispondenza che nasce per proiezione fra due piani, e nella quale ovviamente ai punti ed alle rette dell'un piano corrispondono i punti e le rette dell'altro. La nozione di cor-

(4) Dell'indirizzo medesimo che il Cremona seppe magistralmente sfuttare, già si erano avuti saggi pregevoli con Poncexet, Chasles; Magnus. 
rispondenza o trasformazione è poi stata estesa in vari sensi, e si è dimo* strata altrettanto fondamentale per la geometria quanto quella di funzione per l'analisi. Essa può venir sfruttata da due diversi punti di vista. Il primo utilizza la trasformazione di una figura in un' altra mediante una data corrispondenza, per dedurre dalle proprietà note della prima nuove proprietà della seconda. L'altro punto di vista - di gran lunga più elevato, e posto in rilievo da F. KLEIN (1872) - consiste nel caratterizzare ogni ramo di geometria mediante un gruppo di trasformazioni: così, ad es., la geometria elementare studia le proprietà delle figure che non si alterano per movimenti; mentre la geometria proiettiva si occupa invece di quelle proprietà che restano inalterate di fronte alle trasformazioni proiettive.

Il CRemona si propose pel primo la ricerca delle più generali corrispondenze fra due piani o fra due spazi che mutano punti in punti e rette in curve algebriche, pervenendo così a quelle trasformazioni birasionali che meritamente oggi portano il suo nome. Non già che non si conoscessero prima esempi di tali trasformazioni: ma spetta al Cremond di aver impostata la questione in tutta la sua generalità, risolvendola brillantemente in un modo che - nel caso del piano - può ritenersi completo. Ben si comprende come, addottando il primo dei due punti di vista di cui dianzi ho fatto cenno, questi risultati si prestassero agevolmente ad una numerosa serie di applicazioni : fra le più memorabili ricorderò lo studio delle superficie algebriche dei primi ordini - e, in particolare, delle cubiche - fatto dallo stesso CREMONA, col quale si ricollegano più o meno intimamente ricerche posteriori di Caporali, De Paolis, Bertini, Berzolari, Crani, ece. Il secondo punto di vista doveva condurre più tardi - quando, come vedremo, saranno utilizzabili altri strumenti - alla creazione di una nuova geometria algebrica: quella che studia le proprietà degli enti algebrici che sono invarianti di fronte alle trasformazioni birazionali; ma ad essa il CREMoNa non contribuì in modo diretto.

In pochi anni, mereè l'opera del Cremona, sorgeva in Italia una fiorente Scuola geometrica, che contava fra i discepoli diretti Caporalt, Da Paolis, Veronese, Bertini, Gucoia e Montesano; e vari altri lavoravano con successo nello stesso indirizzo, come p. es. Martinenti e Der Re. Ciò è da ascriversi, oltre alla fecondità e profondità dei metodi e dei risultati del Cremoxa, alla rara comunicativa ed alla potenza organizzativa di quel Grande, in eui - come disse M. Noether $\left({ }^{5}\right)$ - $\mathrm{i}$ geometri di tutti i paesi, e non «per ultimi i tedeschi, riconoscono uno dei loro geniali Maestri ».

(5) «athematische Annalen », t. 5y (1904), p. 1. 
Un'altra Seuola geometrica - notevole par essa, benche non altrettanto numerasa ed originale - fu quella che faceva capo al BATTAGLin, o che, ispirandosi all' opera di Cayley, Sxlvester e Gordan, si occupava principalmente dello studio geometrico degli invarianti e covarianti delle forme algebriche; fra i migliori seguaci di questo indirizzo ricordo D'Oridro, Gerbatid, e - in epoca più recente - Berzolart e Pasoal.

Dovrei infine - per completare il quadro - parlare dell'opera geome. trica di BeLmRaMr: ma di ciò dirò in seguito, quando tratterò della geometria differenziale.

Ho già accennato che la geometria in Italia nell' ultimo quarto del secolo scorso, superato lo stadio meramente proiettivo - e che, in relazione agli sviluppi ulteriori, può dirsi elementare - si andava gradatamente orientando verso nuove vedute e nuovi problemi, aventi la loro essenza nella considerazione del gruppo delle trasformazioni birazionali. Caratteristico a questo proposito è l'elegante risultato del BERTINI (1877-80), che riduce a 4 soli tipi birazionalmente distinti le involuzioni piane del secondo ordine, riguardandosi dunque come identiche due involuzioni piane riducibili l'una all'altra me. diante trasformazioni cremoniane.

Il trapasso verso la concezione puramente birazionale dei problemi non poteva tardare; e lo strumento che dapprima meglio doveva prestarsi allo studio di quei problemi, veniva appunto in quell' epoca approntandosi in Italia, collo sviluppo di un altro ramo di geometria, che tosto doveva condurre ad applicazioni notevoli e svariate. Voglio alludere alla geometria degli iperspaxi o metageometria, che - fondata fin dal 1844 da H. Grassmans in ricerche geniali rimaste per lungo tempo ignorate - già aveva avuto in Germania, Francia ed Inghilterra cultori molteplici $\left({ }^{\$}\right)$.

Non erano però mancati i detrattori che, in base a considerazioni metafisiche ohe oggi ci appaiono stravaganti, avevano impugnata la legittimità della nozione di spazio ad un numero qualunque di dimensioni; legittimità che oggi è pienamente riconoseiuta dal punto di vista matematico non solo, ma anche - attraverso la moderna teoria della relatività - dal punto di vista fisico. Le discussioni sull'argomento servirono almeno a porre in luce che - la geometria non studiando gli oggetti in sè, ma solo le mutue rela-

(6) Dapprima rari Antori - e particolamente Lagrange, Jacobr, Caylex, Srlyester, Schlaefli - avevano più o meno timidamente usufruito di considerazioni iperspaziali in ricerche analitiche; poscia via via si giungeva ad una visione sempre più geometrica dei problemi, mercè l'opera di Plubcher, Riemann, Clebsch, Klein, Jordan, Halphen, SalMON, CLIFFORD, eee. 
zioni fra essi - è lecito sostituire quegli oggetti con altri, purchè tali relazioni restino inalterate. Riflessione questa di fondamentale importanza, suggerita specialmente dai lavori del Plukcker, e che in sostanza già equivale alla con* cezione di una geometria astratta, secondo la quale p. es. le coniche di un piano si posson assimilare ai punti di uno spazio a 5 dimensioni.

Queste idee suggestive e feconde, giunsero in Italia ulteriormente elaborate da $\mathrm{A}$. CAYlex e F. KLEIN, prodncendovi un largo ed importante movimento di stadi e di ricerche. Ed ecco infatti, verso il 1868, il BeLtrame e, verso il 1877 , il D'Ovidio iniziare nel nostro Paese lo studio degli spazi ad $n$ dimensioni, seguiti a breve distanza dal VERonese, il quale - in una Memoria fondamentale (1881) che segna un momento storico nello sviluppo della geometria italiana - edifico sinteticamente la geometria pro* iettiva degli iperspazi (di cui esistevano pochi cenni in un lavoro di CAYLEY del 1846), sulla base del metodo delle proiezioni e sezioni; il Veronese, e più tardi altri, fra cui specialmente Corrado Segre, E. Bentini, G. Lorra e P. Del Pezzo, fecero altresì varie eleganti applicazioni alla geometria ordinaria, mostrando come certe figure del nostro spazio possano talora venir vantaggiosamente considerate quali proiezioni di figure più semplici degli spazi superiori.

Un assestamento definitivo della geometria proiettiva iperspaziale si ebbe in fine attraverso l'opera multiforme di CoRrado Skgre, il quale, da un lato - seguendo de orme del CREMona - seppe opportunamente sfruttare le nozioni ed i risultati che l'algebra deve a Kronecker, WEIERstrass e Frobertus, innestandoli nella trattazione geometrica; e dall'altro - mettendo sistematicamente alla prova la concezione astratta della geometria riusci a cogliere larga messe di risultati ed a fare dello spazio ad $n$ dimensioni l'ambiente naturale in cui si dovevano considerare i fatti geometrici.

Nel suddetto ordine d'idee si inquadra tutta una serie di ricerche di geometria numerativa, di quel ramo cioè che ha per iscopo di determinare il munero delle soluzioni dei problemi che si incontrano nella geometria algebrica, e del quale erano stati fondatori Poncelet, Stenner, Chasces, Jonquikires, Cremona, Schubert, Zeuthen.

Il SEGRE, pur non contribuendo alla geometria numerativa con notevole copia di risultati personali, fu in essa un Maestro e un incitatore, tanto che quasi tutti i numerosi discepoli diretti o indiretti di lui (CAstelndovo, PIERr, Fano, Beppo Levi, Tanturri, Severt, Giambelit, Togitatti, ecc.) hanno lavorato in questo campo, nel quale son da segnalarsi come particolarmente importanti i risultati del SEVERI, relativi all' estensione del metodo funzionale di CAYLEY, ai caratteri proiettivi ed alle intersezioni delle varietà algebriche superioni, ed alla validità del prineipio della conservazione del numero. 
L'attività di CoRrado Segre come Scienziato e come Maestro - - secondo avrò occasione di precisare nel seguito - ebbe anche un'inflnenza decisiva in altri rami della geometria; per ora mi limito a segnalare l'importante ufficio che essa ebbe nell' elaborazione per via iperspaziale - avvenuta verso il 1890 - della nuova teoria delle curve algebriche, caratterizzata dal gruppo delle trasformazioni birazionali, ed alla quale presero altresi parte attiva il Bertint ed il Castelnuovo. Detta teoria, sorta dalle immortali ricerche di BERnaRdo RIEMANN intorno alle funzioni algebriche di una variabile ed ai loro integrali - acutamente sviscerate, da un punto di vista più geometrico, dal CLEBsch - e dai profondi sviluppi algebrici di BRILL e NoETHer, veniva così completata dalle ricerche suddette, sino a formare un tutto unico - che qui neppur posso adombrare - ove sono elegantemente congiunti i tre suoi aspetti trascendente, algebrico e geometrico, e nel quale s'inquadrano le classiche ricerche trascendenti di HuRwitz e quelle algebricogeometriche di SEVERI sulle corrispondenze.

Rielaborata così la teoria invariantiva delle curve algebriche in modo che può ritenersi definitivo - e che ha ricevuto assetto in recenti Trattati italiani, da tutti conosciuti - la geometria italiana si volse alla tem.ia invariantiva delle superficie algebriche, sempre dal punto di vista delle trasformazioni birazionali. I fondamenti di tale teoria erano stati gittati all'estero, sopratutto attraverso la grande opera di MAX NoETHER. Risultati importanti si possedevano anche in seguito ai lavori di CLEBsch, CAYLEY, ZEuthen, PICARD, per ciò che concerne le superficie qualunque, e degli scolari diretti e indiretti del Cremona (Bertini, Caporali, Del Pezzo, Guocta, Jung, Marminetri, ecc.), per cio che concerne le superficie razionali e i sistemi lineari di curve piane, la cui teoria era stata esposta e completata in vari punti essenziali in un bel lavoro del CAstelnuovo.

Ma tutto questo lasciava presentire le difficoltà gravissime che avrebbe offerto l'inquadramento sistematico dei risultati noti in un' ampia teoria organica, e la risoluzione dei molti problemi elevati e difficili, che occorreva tuttavia di sciogliere.

Quel che in proposito occorreva fu fatto; e fu fatto in Italia in due distinti periodi: il primo dei quali va dal 1893 al 1900 ed è precipuamente dominato dalle idee del Castelndovo e dell' ENRIQUEs; il secondo comincia col 1904 ed è nettamente dominato dalle idee del SEvEr. I due quadrienni 1896-1900 e 1904-1908 rappresentano per così dire i momenti eroici della mirabile e grandiosa costruzione.

Nei lavori di Castelnuovo ed EnRIQUes giuoca principalmente la considerazione dei successivi aggiunti di un dato sistema lineare (considerazione che - in casi particolari - già era stata sfruttata da KANToR); inoltre 
- pur non venendo completamente abbandonate le vedute iperspaziali del SEgRE - in essi diviene più nitida e più largamente operativa la concezione invariantiva dei problemi, distinguendosi chiaramente fra invarianza assoluta e relativa, ed andando in fondo nella spinosa questione delle cosi dette curve eccezionali, già considerate nebulosamente dal NoETHER. Al SEvERI debbonsi fra l'altro varie nozioni dimostratesi nella teoria di importanza fondamentale -- come quelle di curva virtuale, e di serie caratteristica d' un sistema continno di curve su di una superficie algebrica - nonchè la geniale fusione dei metodi algebrico-geometrici coi metodi analitico-trascendenti, che erano stati coltivati in Francia dal Poincaré, dal PICARD e dall' Humbert; e la teoria della base con eui egli crea un'algebra delle curve sopra una superficie algebrica, che ha avuto ripercussioni notevoli anche nello sviluppo della topologia, Al SEvERI si debbon inoltre i fondamenti della geometria sulle varietà algebriche a più dimensioni.

L'edificio grandioso - onore e vanto della scuola geometrica italiana che in tal guisa si è venuto costruendo, anche per merito di FANo, BEnzo. lari, Beppo Levi, De Franohis, Bagnera, Scorza, Chistni e dei discepoli diretti o indiretti del Severi - Rosati, Torelli, Comessatri, Albanese e vari altri, fra i quali io stesso - non può venir delineato in pochi tratti; mi limiterò dunque a dire che in esso si fondono armonicamente teorie elevatissime e disparate, con uno stile caratteristico, di classica bellezza, e che la difficile questione della risoluaione delle singolarità delle superficie fu sciolta dal nostro illustre Collega BePPo LEvI (?). Non senza rammarico mi tocea per ultimo osservare che - mentre all' estero vari sono i matematici che attualmente si interessano a questioni che più o meno intimamente si collegano a questa splendida tradizione geometrica $\left({ }^{8}\right)$ - non sono molti fra noi i giovani che la continuano. Che, se è vero che le principali questioni rimaste insolute appaiono assai difficilmente sormontabili, ciò anzi dovrebbe costituire un' ot. tima ragione per cimentarvisi, in chi - rifuggendo dai facili successi aspiri veramente a lasciare un' orma personale nella scienza.

Un'altra via maestra, seguendo la quale gli Italiani si son pure brillantemente affermati, è quella della geometria differensiale. Questa disciplina

(7) Ricorderò ancora i classici risultati di CASTELxuovo sulla razionalità cielle involu* zioni piane e sulla caratterizzazione delle superficie razionali; quelli di ENaıues sulle superficie di genere geometrico nullo, di GNRIQUES, FaNo e SEvERI sulle superficie con infinite trasformazioni birazionali in sè e di ENRIQUES e SEverI sulle superficie con curva canonica d'ordine zero; ed infine le fondamentali ricerche di Enriques, Severi, Bagnera e De Francuis sulle superficie iperellittiche.

(8) Basti fare i nomi di Lefschetz, Snyder, Godeaux, Rosenblate, Zariski. 
- che occupa per così dire una posizione strategica fra l'analisi e la geometria, e le cui più remote origini si possono far risalire ad ARchnmene -sorge contemporaneamente al calcolo infinitesimale, per tosto elevarsi a scienza in certa guisa autonoma, mercè l'opera di Euler, Monge, Gauss e dei loro seguaci, ai quali si debbon lo studio delle prime proprietà relative alla curvatura di curve e superficie, e delle questioni concernenti le congruenze di raggi, le coordinate curvilinee, la costruzione delle carte geografiche e l'applicabilita fra superficie flessibili ed inestendibili.

Non v'era fra noi, prima della proclamazione del Regno d'Italia, alcuna cattedra da cui quella disciplina potesse esser divulgata; tuttavia i rudimenti venivano allora generalmente insegnati nei Corsi di calcolo Igratificati in quei tempi dall'aggettivo di sublime), e non mancavano cultori appassionati, come i già menzionati MAINARDI e BondoNI, ai quali occorre agginngere Delfino Codazzi, più tardi egli pure professore a Pavia, che - in una Memoria presentata nel 1859 all'Accademia di Francia - diēe le celebri formule che ora portano il suo nome unitamente. a quello del MAINARDI, che le aveva ottenute prima. Dopo il '60, dette teorie trovarono il posto che loro spettava nei Corsi di geodesia, e ricevettero contributi essenziali da parte di Scienziati italiani.

Chi prineipalmente afferrò l'importanza e la fecondità delle idee di Gauss, riuscendo in breve a diffonderle fra noi, fu Francesco Brioschi; e dalla sua Scuola uscì Eugenio BeLtram, che - dopo aver insegnato durante l'anno scolastico 1862-63 algebra e geometria analitica qui in Bologna - sali alla cattedra di geodesia dell' università di Pisa, chiamatovi dal Bextr. Durante il triennio trascorso a Pisa, l'attività del Beurram si volse con pieno successo alla geometria differenziale, ispirandosi specialmente alle vedute di Bernardo Riemann, il quale, per motivi di salute, aveva fissato la sua dimora in quella città. $\mathrm{E}$, in breve volger di tempo, divennero classici i risultati del BeLtrami relativi alla rappresentazione geodetica delle superficie, ai parametri differenziali, alle variabili complesse, alle superficie di area minima, agli spazi di curvatura costante, ed a quella luminosa interpretazione della geometria non enclidea, che venne inaspettatamente a chiarire una controversia allora agitata intorno ai principi della geometria.

Ha così origine tutta una serie di ricerche, che comprende la prima attività del DrNI, al oui nome restò legata una particolare superficie a curvatura costante negativa; quella del CesARo -... relativa alla geometria in. trinseca - improntata a rara eleganza e densa di contenuto geometrico; quella del RIoor, che - col crlcolo assoluto, al quale pure contribuì pode. rosamente il Levi-Cryita - creó uno degli strumenti essenziali di cui si è 
poi valso Erastedn nella sua teoria della relatività; per eulminare infine con l'opera grandiosa di Lurgr Branchr e della sua Seuola, che valse a far progredire notevolmente quasi tutti i capitoli della geometria differenziale.

Una delle idee più feconde che debbonsi al BIANoHr è quella dell'impiego di opportune trasformazioni, quale ad esempio quella che fa passare dall' una all'altra delle due falde focali di una conveniente congruenza di raggi o di sfere. Di tale tipo \& la celebre trasformanione complementare, che permette - nota che sia una superficie pseudosferica - di dedurne infinite altre. La reiterata applicazione di questa trasformazione, conduce così a sistemi di superficie pseudosferiche dipendenti da un numero grande a piacere di pan rametri; e, dal punto di vista analitico, porge un nuovo strumento per lo studio di determinate equazioni a derivate parziali, reso più potente da elegantissimi teoremi di permatabilitò. L'indirizzo di ricerche in tal guisa inaugurato, ed al quale tosto si associarono attivamente matematici stranieri eminenti quali il LIE, il BaEckLdnd ed il DARBoux, condusse il BIANoHI ad edificare le teorie ormai classiche relative alle superficie a curvatura costante, alle deformate per applicabilitì delle quadriche ed a taluni sistemi tripli ortogonali e tripli coniugati di superficie. Notevoli anche furono gli apporti del BIANOHI alla geometria differenziale non euclidea, e l'abilitì con cui egli seppe dedurne proprietà di geometria euclidea. Numerosissimi i suoi scolari diretti ed indiretti, fra cui Fubint, E. E. Levi, Calapso, Tortorici, Tonolo e Sbrana.

Un ulteriore importantissimo ramo di geometria differenziale, è quello che - fondandosi sulle profonde concezioni e sui risultati del RIEMANy relativi agli spazi curvi - si è venuto rapidamente sviluppando in questi ultimi anni, in vista specialmente delle applicazioni alla teoria della relatività.

Il germe del nuovo larghissimo movimento d'idee risiede nella geniale nozione di parallelismo, introdotta dal Levr-CrviTa nel 1917, mercè la quale si effettua il trasporto di una direzione spiccata da un punto di uno spazio di Ruemani, quando il punto si muova in esso arbitrariamente. Da qui ebbero subito origine numerosissime ricerche, quali quelle di SEveri, BoMPIANI in Italia, e quelle di WeyL, CARTAN, SCHouter all' estero, che portarono alla bella teoria delle connessioni, relativa allo spazio concepito nel modo più generale come una riunione di celle infinitesime - fra loro opportunamente connesse - in ciascuna delle quali sussiste una data geometria (metrica, affine o proiettiva).

A tale teoria - che alla sua volta intimamente si ricollega alla geo. metria proiettiva dei cammini di Veblen, Thomas ed EISEnhart - ha pure contribuito ENEA BORTOLOTTI, il quale fra l'altro ha rilevato una no. 
tevole relazione fra essa $\theta$ l'ordinaria geometria delle varietà immerse in ambiente proiettivo.

Quest' nltima geometria, ossia lo studio delle proprietà infinitesimali delle figure che sono invarianti di fronte alle trasformazioni proiettive, costituisce oggidì un corpo estesissimo di dottrine - più noto col nome di geometria mroiettivo-differentiale - sviluppatosi in gran parte per merito di Geo. metri italiani.

Principalmente due sono gli indirizzi che qui si riscontrano. Il primo. originato dalle ricerche di Halphen, WLlozynski. SANNia e Berzolari, riconduce sostanzialmente lo studio alla determinazione di certi invarianti e covarianti di determinate equazioni alle derivate ordinarie, o di sistemi d'equazioni alle derivate parziali del $2^{\circ}$ ordine. Esso ha recentemente ricevuto una sistemazione definitiva colle fondamentali ricerche del FuBIxr, il quale - mediante la sagace introduzione di opportune forme differenziali, e l'im* portante nozione di deformazione proiettiva - ha potuto ottenere luminosi raffronti coll'ordinaria geometria differenziale metrica, e completare la teoria in vari punti essenziali.

Il secondo indirizzo ha ricevuto la sua caratteristica impronta, prevalentemente sintetica, dalle ricerche di CorRado Smgre relative a questo argomento - precedute da qualche saggio di Dec Pezzo - e conta fra i suoi migliori cultori il Terracini ed il Bomprant. Per quanto in certe questioni i metodi impiegati da questi Autori non possano penetrare così addentro come i precedenti, pure essi hanno un valore altamente suggestivo, poichè si fondano su d'uno studio diretto degli enti geometrici - quasi sempre considerati negli iperspazi - evitando i lunghi calcoli collo sfuttare in sommo grado l'intuizione geometrica. Vari dei risultati conseguiti in quest'ordine d'idee hanno anche importanza per l'analisi, come ad es. quelli sulle così dette reti, che ricevono un'immediata interpretazione nella teoria delle equazioni di LAPLACE. Occorre inoltre, per poter valutare appieno la portata di quelle ricerche, rilevare che molte di esse costituiscono un importante anello di congiunzione fra la geometria differenziale e la geometria algebrica $\left({ }^{9}\right)$.

(9) Numerosissimi sono coloro che all'estero si sono oceupati dei diversi rami di geomotria differenziale. Oltre a quelli gin ricordati nel testo, vanno particolamente monzionati Dupin, Binet, Lamé, Moutard, Jacobt, Minding, Bonnet, Serret, Liomville, Bertrann.

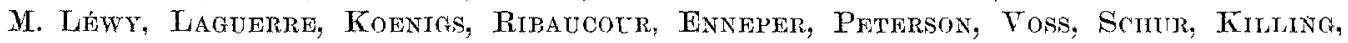
Schwarz, Kyoblatch, Whincartzan, Lipschitz, Christoffei, Scheffers, Lilienthal,

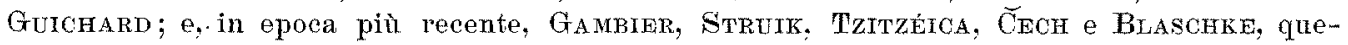
st' ultimo capo di una fiorente Scuola geometrica tedesca. 
Dirò infine brevemente di quel potente mezzo di sintesi geometrica che è il moderno calcolo vettoriale. Sorto dalle ricerche di BeLlavitrs, Grasssmann, Hamilton, Heaviside, ece, esso vanta fra noi numerosi cultori, quali Peano, Burali-Forti, Boggio, Marcolongo ed il nostro illustre Collega Burgatri, ai quali deve contributi notevoli - come ad es. lo studio approfondito delle omografie ed iperomografie vettoriali - ed applicazioni pregevolissime alla geometria differenziale ed a vari altri rami delle matematiche.

Qui arresto la rapida esposizione, di cui non mi nascondo le deficienze e le lacune, in parte dovute alla ristrettezza del tempo. Non ho, ad esempio. potuto parlare delle ricerche sui fondamenti della geometria di VERONESE, Peano, Preri, Enriques, Fano, Beppo Levi (1i), e del grandioso movimento filosofico che - ricollegandosi ad esse - ha portáto alle più importanti concezioni della relativita; neppure ho detto della topologia o amalysis situs, benchè, per dirla col SEverI $\left({ }^{11}\right)$ - alla cui efficace attività si deve la diffusione fra noi dei risultati conseguiti negli ultimi anni all' estero in questo campo - l'Italia abbia avuto nel BETTI ed in vari cultori di geometria algebrica, rispettivamente l'antesignano e gli apostoli dell'importanza di tali studi: ed anche ho taciuto di quella geometria ipercomplessa che - originata da ricerche di CORRADo SEgRE rimaste in ombra per lungo tempo ha recentemente trovato applicazioni notevoli e svariate (iz).

Per dare un'idea completa del periodo storico che ho voluto tratteggiare, avrei poi dovuto porre meglio in Ince i legami col movimento scientifico delle altre Nazioni, e cogli altri rami della scienza. A questo proposito mi limito a constatare che l'antagonismo che ancor non molti anni fa sussisteva fra Analisti e Geometri, è oggi completamente superato. "Analisi e geometria » osserva il Volterra $\left({ }^{1 *}\right)$ « che furon ritenuti ed impiegati come due termini «opposti, non possono, nè per la loro origine, nè per la loro storia, nè per «la natura loro, farisi corrispondere a concetti che si eliminino e si escla«dano a vicenda; dirò anzi che non possono porsi a confronto, come non «può stabilirsi un rapporto fra il colore ed il volume, fra il peso e la forma « dei corpi ».

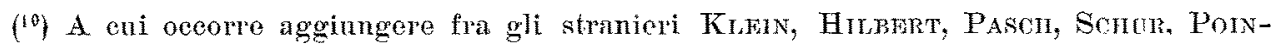
CARÉ, SCHOWNFLIES.

(11) F. Styerr, Moderni indirizzi nelle matematiche. "Atti della Socielà Italiana per il Progressi delle Scienze " XVII Riunione, Settembre 1928).

(12) Specialmente per merito di Severi e Cartan.

(13) Loc. eit. in (1), p. 62. 
La distimzione fra analisi e geometria ò, secondo me, analoga a quella che intercede fra un'opera di musica ed una di scultura; ambedue agiscono sul nostro intelletto soddisfacendo a quel sentimento estetico - insito in noi - che ricerca nelle cose l'armonia e la proporzione, l'ordine e la misura: l'una trae la sua éssenza dal concetto di numero il quale - secondo Platone - governa il mondo, e l'altra da quel senso plastico così vivo nell' nomo da far dire allo stesso Platone che Dio eternanente geonetrizza. $\mathrm{Ma}$, come gli antichi vedevano la suprema bellezza del creato nell' armonia delle sfere, così non si può penetrare lo spirito di ciò che ̀̀ oggi la matematica, senza una visione d'assieme che permetta di apprezzare l'unità monolitica del maestoso edificio. Tanto più che i ravvicinamenti sono essenziali al progresso della scienza; e varie delle più recenti teorie non si posson neppure nettamente assegnare all'analisi piuttosto che alla geometria o viceversa. Valga come esempio la perspicua geometria dello spazio hilbertiano, colla quale l'illustre Collega VITALI ha potuto assoggettare all'intuizione geometrica uno dei più elevati capitoli di analisi - il calcolo funzionale - originato dai magistrali lavori del Pinoherce e del Voltrerra.

Il quadro da me delineato - ancorchè imperfetto - non può non col. pire per la multiforme ricchezza e la vastità degli argomenti. Dei numerosi rami che si dipartono dal rigoglioso tronco della geometria, formerò oggetto di successivi Corsi di geometria superiore. Principiero intanto colla geometria algebrica, che più profondamente porta impressa l'orma del genio italiano, riattaccandomi intimamente alla tradizione del CREMonA, la quale non può nè deve morire. Udite le parole di entusiasmo e di fede, pronunciate dallo stesso CREmona nella chiusa smagliante della sua citata Prolusione $\left({ }^{14}\right)$.

« Respingete da voi, o giovani, le malevole parole di coloro che a con«forto della propria ignoranza o a sfogo d'irosi pregiudizi vi chiederanno « con ironico sorriso a che giovino questi ed altri studi, e vi parleranno del. «l'impotenza pratica di quegli uomini che si consacrano esclusivamente al «progresso di una scienza prediletta. Quand' anche la geometria non rendesse, « come rende, immediati servigi alle arti belle, all'industria, alla meccanica, "all'astronomia, alla fisica; quand' anche un'esperienza secolare non ci am«monisse che le più astratte teorie matematiche sortono in un tempo più o «meno vicino applicazioni prima neppur sospettate; quand'anche non ci «stesse innanzi al pensiero la storia di tanti illustri che senza mai desistere « dal coltivare la scienza pura, furono i più efficaci promotori della presente

(14) Op. oit. in (2), p. 253. 
«civiltà - ancora io vi direi: questa scienza d degna ohe voi l'amiate; "tante sono e così sublimi le sue bellezze ch'essa non può non esercitare «sulle generose e intatte anime dei giovani un'alta influenza educativa, «elevandole alla serena e inimitabile poesia della verita! I sapientissimi " antichi non vollero mai scompagnata la filosofia, che allora era la scienza «della vita, dallo studio della geometria, e PLATone seriveva sul portico "della sua accademia: Nessuno entri qui se non è geometra. Lungi dunque « da voi questi apostoli delle tenebre; amate la verità e la luce, abbiate fede " nei servigi che la scienza rende presto o tardi alla causa della civiltà e «della libertà. Credete nell'avrenirel questa è la religione del nostro secolo».

La passione travolgente che animava il CRemona, e ch'egli seppe in modo così efficace trasfondere nei suoi discepoli, pare - s'io non m'inganno - oggi vada leggermente smorzandosi. Diminuito è fra noi il numero dei giovani che si dedicano alla scienza pura, e molti di questi si volgono di preferenza verso le scienze fisiche, attratti forse da ricerche che sembrano avere un maggior interesse pratico, e da quelle recenti vedute che son venute a sconvolgere molte delle nozioni che per l'addietro si ritenevano stabilmente acquisite.

Si rammenti però - col JACOBr $\left({ }^{(5)}\right)$ - che l'unico scopo della scienza è l'onore dello spirito umano; e, da questo punto di vista, una questione sui numeri equivale ad una questione sul sistema del mondo. Aggiungasi - ammonisce il Severri $\left({ }^{15}\right)$ - che $\ll$ il giorno che decadesse il livello dei "nóstri studi matematici sarebbe un bruttissimo giorno per la Nazione, in «quanto, a scadenza più o meno lunga, ne deriverebbe un decadimento del "livello scientifico generale ed in modo particolare quello delle scienze fi«siche. Ricordiamoci che Napoleone considerava l'alto sviluppo degli studi «matematici legato alla grandezza e alla prosperita della Nazione; e che lo «stesso concetto napoleonico ha ispirato la Germania nel periodo più bril= «lante della sua ascensione».

Possa, o giovani, il mio insegnamento contribuire a dischindervi le arcane bellezze delle matematiche diseipline, o ad iniziare qualcuno di voi alle dolcezze sublimi della ricerca scientifica, paragonate dal JACOBI ( ${ }^{17}$ ) al magico

(15) Lettera a LEGENDRE (2 luglio 1830); cfi. C. G. J. JACOBI, Gesammelte Werke, t. I (Berlin, 1881). p. 453 .

(16) Nel Rapporto tenuto alla XIX riunione della Società Italiana per il Progresso delle Scienze (Settembre, 1930).

(17) Cfr. Kronecker, Ueber den Zahlbegriff, "Journal für Math. », t. 101 (1887), p. 337. 
effetto che -- secondo la narrazione di Oukro - producevan sui messi di Ulisse i fiori del loto:

"Chiunque l'osea dilettosa e nuova

"Gustato avea, con le novelle indiewo

« Yon bramava tornar: colả bramava

* Starsi e, mangiando dol soave loto,

"La contrada natia sbandir dal petto" (18).

Non già però - come così verrebbe a significare l'ultimo verso preso alla lettera, e come alle volte erroneamente si opina - che lo Scienziato sia quasi estraneo alle contingenze materiali della vita: poichè anzi - attesta la storia - l'amor di patria è sempre stato una delle caratteristiche sue più spiccate; e dai suoi studi astratti egli ha saputo trarre sovente una comprensione della vita più suggestiva e profonda.

E non crediate neppure che il possesso della scienza sia agevole quanto quello dei frutti del loto! Esso esige per contro costanza e abnegazione e spirito di sacrificio grandi, quali solo posson esser dettati ad una mente eletta, dall'amore per la scienza e dalla fede incrollabile nella continua ele. vazione dello spirito verso uno stato di perfezione sublime, che - per quanto forse irraggiungibile - costituisce la meta ideale in cui armoniosamente si fondono il Buono ed il Bello. Ed è appunto il faticoso sforzo ehe compie per procedere su questa via - unito all' appagamento del più raffinato senso estetico - che procura allo studioso soddisfazioni purissime, analoghe al senso di gioia che anima l'alpinista nella conquista della vefta.

(15) Iliade, eanto $\mathrm{IX}$. 